\title{
Der Wissenschaftsbegriff der Psychotherapiewissenschaft
}

\author{
Markus Erismann
}

\author{
Psychotherapie-Wissenschaft 9 (2) 13-17 2019 \\ www.psychotherapie-wissenschaft.info \\ CC BY-NC-ND \\ https://doi.org/10.30820/1664-9583-2019-2-13
}

\begin{abstract}
Zusammenfassung: Zwar ist eine wissenschaftliche Praxis ohne Wissenschaftsbegriff möglich. Der Wissenschaftsbegriff einer bestimmten Wissenschaft konstituiert und klärt aber das Selbstverständnis dieser Wissenschaft. Die Absicht meines Artikels ist die Darstellung und Diskussion des Wissenschaftsbegriffs der Psychotherapiewissenschaft. Dieser weicht vom allgemein anerkannten Wissenschaftsbegriff ab: Er bezieht das Subjektive mit ein, legt den Fokus auf das Individuelle und erkennt Widersprüche und Ambivalenzen als Faktoren wissenschaftlicher Erkenntnis an. Ich will zeigen, wie trotz dieser Abweichungen eine Übereinstimmung zwischen dem Wissenschaftsbegriff der Psychotherapiewissenschaft und der allgemein anerkannten Auffassung von Wissenschaft möglich ist.
\end{abstract}

Schlüsselwörter: Wissenschaftsbegriff, Psychotherapiewissenschaft, das Subjektive, das Individuelle, das Widersprüchliche

\section{Einleitung}

Die Frage nach dem Wissenschaftsbegriff einer Wissenschaft ist die Frage nach deren Verständnis von Wissenschaft überhaupt und deren Selbstverständnis als Wissenschaft. Sie stellt sich in Phasen der Krise einer Wissenschaft oder in einer Phase, in der diese Wissenschaft noch nicht den Zustand eines Paradigmas bzw. einer anerkannten Normalwissenschaft erreicht hat. Gemäss Fischer (2008, S. 1) befindet sich die Psychotherapiewissenschaft noch in einer «prä-paradigmatischen Phase».

Zwar ist wissenschaftliche Praxis ohne Wissenschaftsbegriff möglich. Der Wissenschaftsbegriff einer einzelnen Wissenschaft dient aber der Bildung und Klärung von deren Selbstverständnis und ist ein Element ihres wissenschaftstheoretischen Rahmens. Die Absicht meines Artikels ist die Darstellung und Diskussion des Wissenschaftsbegriffs der Psychotherapiewissenschaft. Dessen Ausarbeitung soll das Bemühen der Psychotherapiewissenschaft um Etablierung als eigenständige und gleichwertige Disziplin an der Seite von Psychologie und psychiatrischer Medizin unterstützen. Der spezielle, schulenübergreifende Wissenschaftsbegriff der Psychotherapiewissenschaft soll in ein Verhältnis zu Aspekten eines allgemeinen Wissenschaftsbegriffs von Natur- und Geisteswissenschaften gesetzt werden.

Zuerst werde ich einige Aspekte eines allgemeinen Wissenschaftsbegriffs diskutieren. Dabei werden drei Charakteristika der Psychotherapiewissenschaft sichtbar, die dem allgemeinen Wissenschaftsbegriff scheinbar widersprechen. Die Frage stellt sich, inwiefern trotz dieser dem allgemeinen Wissenschaftsbegriff widersprechenden Charakteristika eine Konkordanz zwischen dem allgemeinen und dem psychotherapeutischen Wissenschaftsbegriff hergestellt werden kann. Für die Psychotherapiewissenschaft wird ein Wissen- schaftsbegriff in der Schweizer Charta (2016) expliziert, den ich kommentiere. Anschliessend werden die drei dem allgemeinen Wissenschaftsbegriff widersprechenden Charakteristika der Psychotherapiewissenschaft diskutiert. Schliesslich soll unter Berücksichtigung des Aufsatzes von Wagner (1996) das wissenschaftliche Selbstverständnis der Psychotherapie in ein Verhältnis zu dem der Medizin, Psychiatrie und klinischen Psychologie gesetzt werden.

\section{Aspekte eines allgemeinen Wissenschaftsbegriffs}

Angesichts der Verschiedenartigkeit dessen, was unter «Wissenschaft» verstanden wird, besteht der Ausweg «in dem, was dieses Verschiedenartige miteinander verbindet, also in den Gemeinsamkeiten der unterschiedlichen Wissenschaftsauffassungen» (Wohlgenannt, 1969, Vorw.). Die folgende Darstellung einiger Aspekte eines allgemeinen Wissenschaftsbegriffs benennt wissenschaftliche Grundelemente und Kriterien für Wissenschaftlichkeit.

Der Mediziner und Wissenschaftstheoretiker Diemer (1964, S. 67) formulierte den allgemeinen Wissenschaftsbegriff wie folgt: «Wissenschaft ist ein Gesamt von Sätzen über einen thematischen Bereich, die mit diesem in einem Begründungszusammenhang stehen.» Dabei geht es um begründete und wahre Sätze. Begründung und Wahrheit sind miteinander verbundene Kriterien wissenschaftlicher Sätze: Begründet wird die Wahrheit der Sätze, das heisst ihre Übereinstimmung mit etwas Gegebenem. Diese Übereinstimmung muss überprüfbar sein. «Begründungszusammenhang» meint daher bei Diemer die begründete und überprüfbare Übereinstimmung zwischen Sätzen bzw. Aussagen mit einem gegebenen, thematisierten Sachverhalt. 
Die Sätze, die Wissenschaftlichkeit für sich beanspruchen, müssen bestimmten, fundamentalen Wissenschaftskriterien genügen. Grundlegende Kriterien sind: Objektivität (Begründung und Wahrheit), Überprüfbarkeit (Verifizierbarkeit bzw. Falsifizierbarkeit) und Intersubjektivität (Verständlichkeit und Nachprüfbarkeit). Diese Triade eng miteinander verbundener Kriterien bildet die Grundlage für relativ sicheres Wissen.

Hinsichtlich dieses Zusammenhangs kann etwa die Frage nach der Subjektivität der Wissenschaft gestellt werden. So gibt es in der Biologie und der psychosomatischen Medizin die Idee der «Einführung des Subjekts» (v. Uexküll, 1931) oder in der Psychotherapiewissenschaft des «Einbezugs des Subjektiven» (Schweizer Charta, 2016) in den wissenschaftlichen Prozess. Die Subjektivität steht im Widerspruch zur genannten Triade und damit zum allgemeinen Wissenschaftsbegriff. Zu klären ist, wie der «Einbezug des Subjektiven» zu verstehen ist. Darauf soll nachfolgend noch eingegangen werden.

Wissenschaft besitzt zudem einen Allgemeinheitscharakter. Frank (1991, S. 51) vermutet, «dass das Projekt der Wissenschaftlichkeit im Prozess abendländischer Rationalität geradezu über einem Ausschluss von Individualität errichtet wurde. Schliesslich wäre wissenschaftliches Arbeiten ohne eine gewisse Option aufs Allgemeine undurchführbar.» Gesucht werden in der Wissenschaft Regularitäten, Muster, Strukturen und Gesetze, in denen das Individuelle und Besondere durch Subsumption unter das Allgemeine gleichsam verschwindet.

Ein weiteres Kriterium für Wissenschaftlichkeit ist die Forderung nach Widerspruchsfreiheit bzw. Konsistenz. Eine Gesamtheit von Sätzen bzw. Aussagen darf nicht zwei einander widersprechende Aussagen oder sich selbst widersprechende Aussagen enthalten. Die Aussagen müssen den Regeln der Logik gehorchen, die keine Widersprüche erlaubt. Treten dennoch Widersprüche auf, sind sie zu eliminieren. Inwiefern mit diesem, Widersprüche ausschliessenden Kriterium dialektisches Wissen als unwissenschaftliches zu kennzeichnen wäre, soll ebenso nachfolgend diskutiert werden.

Die genannten Kriterien für Wissenschaftlichkeit sind durch einen dreifachen Ausschluss charakterisiert, der nicht zuletzt im Hinblick auf den Wissenschaftsbegriff der Psychotherapiewissenschaft von Relevanz ist. Es ist dies der Ausschluss des Subjektiven, des Individuellen und des Widersprüchlichen. Dieser Ausschluss wird nun dahingehend diskutiert werden, inwiefern trotz Einbezug des Subjektiven, des Individuellen und des Widersprüchlichen seitens der Psychotherapiewissenschaft eine Konkordanz mit dem zuvor explizierten allgemeinen Wissenschaftsbegriff hergestellt werden kann.

Die Antwort auf die Frage nach dem Wissenschaftsbegriff einer Wissenschaft ist eine zweifache: 1) das Verständnis dieser Wissenschaft von Wissenschaft überhaupt; 2) das Selbstverständnis dieser Wissenschaft als Wissenschaft. Beide Auffassungen müssen, damit die Wissenschaft Anerkennung findet, mit dem allgemein anerkannten Wissenschaftsbegriff konkordant sein.

\section{Das Verständnis der Psychotherapiewissenschaft von Wissenschaft überhaupt}

\section{Systematik und Methode}

Eine Antwort hinsichtlich des Verständnisses der Psychotherapiewissenschaft von Wissenschaft überhaupt findet sich in der Schweizer Charta (2016, Abs. 2.8): «Im Sinne dieser Charta verstehen wir unter wissenschaftlich ein systematisches, methodisches Arbeiten, das der Entwicklung der Theorie oder der Vermittlung zwischen Theorie und klinischer Praxis dient.» Hier werden das Systematische und das Methodische als Kriterien für Wissenschaftlichkeit genannt, eine Auffassung, die einem allgemeinen, wissenschaftstheoretischen Konsens bezüglich Wissenschaftlichkeit entspricht.

Das System als geordnete Gesamtheit und die Methode als ordnungsstiftendes Verfahren bilden zwei Dimensionen der Wissenschaft. Das Systematische und das Methodische sind zwei Hinsichten, auf die sich wissenschaftstheoretische Reflexion bezieht. Unter WissenschaftstheoretikerInnen besteht allerdings keine Einigkeit darüber, welcher dieser beiden Dimensionen Priorität zukommt.

Für Buchmann und Kollegen (1996, S. 105) ist es die besondere Forschungsmethode, die der Psychotherapiewissenschaft ihre Eigenständigkeit verleiht: «Dieses eigenständige Vorgehen (Methode) zur Untersuchung und Datenerhebung während der Interaktion durch den Therapeuten bei sich selber, beim Patienten und hinsichtlich des interaktiven Prozesses, macht die Psychotherapie zur eigenständigen Wissenschaft.»

\section{Die dem allgemeinen Wissenschaftsbegriff widersprechenden Charakteristika der Psychotherapiewissenschaft}

Was das Systematische und das Methodische als wissenschaftliche Kriterien anbelangt, besteht eine Konkordanz mit dem allgemeinen Wissenschaftsbegriff. Dagegen widerspricht 1) der «Einbezug des Subjektiven» der Triade von Objektivität, Überprüfbarkeit und Intersubjektivität, 2) die Fokussierung auf das Individuum der allgemeinen wissenschaftlichen Ausrichtung auf das Allgemeine, sowie 3) der Einbezug des Widersprüchlichen der allgemeinen Forderung nach Widerspruchsfreiheit. Im Folgenden soll anhand der Analyse der drei genannten Aspekte gezeigt werden, wie trotz dieser scheinbaren Unvereinbarkeiten zwischen dem allgemeinen Wissenschaftsbegriff und dem Wissenschaftsbegriff der Psychotherapiewissenschaft eine Konkordanz möglich ist.

1) Gegenüber der "Austreibung des Subjekts aus der modernen Medizin» geht es in der psychosomatischen Medizin «von Anfang an um die Widereinführung des Subjekts» (Wagner, 1996, S. 225). Derselbe wissenschaftstheoretische Vorgang findet sich in der Psychotherapiewissenschaft mit dem «Einbezug des Subjektiven» gegenüber der klinischen Psychologie und deren «Bewertung von Subjektivem als Störfaktor» (ebd., S. 227). Der Subjek- 
tivität in der Wissenschaft, die doch auf Objektivität aus ist, haftet ein Moment der Willkür an. Die Einführung des Subjektiven in den wissenschaftlichen Prozess ist nur dann möglich, ohne dass das Moment der Willkür bestimmend wird, wenn das Subjektive mit kritischer Reflexion einhergeht. Gerade dieser «systematische und kritisch reflektierte Einbezug des Subjektiven» gehört für Buchmann und Kollegen (1996, S. 105) «zum wesentlich Eigenen der Wissenschaft Psychotherapie».

Psychotherapie als beziehungsorientiertes Heilverfahren basiert per se "auf einem intersubjektiven Geschehen" (Schweizer Charta, 2016, Abs. 1.8), bewegt sich also immer schon innerhalb der Dimension der Intersubjektivität. Das Moment der Willkür, das mit dem Subjektiven verbunden ist, lässt sich im psychotherapeutischen Prozess minimieren bzw. aufheben, indem das Subjektive durch (Selbst-)Reflexion auf die Ebene der Intersubjektivität gehoben wird:

«Intersubjektivität - verstanden als Fähigkeit zur empathischen Perspektivenübernahme und sozialen Kognition als Grundmodell der sozialen Interaktion - setzt eine spezifische Form des selbstreflexiven Selbstbewusstseins voraus. [...] Subjektives Erleben und die Wahrnehmung der (objektiven) äusseren Realität müssen auf einer höheren Ebene integriert werden, um die Stufe der Intersubjektivität zu erreichen» (Barwinski, 2017, S. 23).

Wie verhält sich die Intersubjektivität als Dimension des psychotherapeutischen Beziehungsgeschehens zum allgemeinen Wissenschaftskriterium der Intersubjektivität, die das in der therapeutischen Praxis generierte Wissen betrifft und dessen Objektivität gewährleisten soll? Es ist «die kritische Reflexion der Therapeut/innen bezüglich des eigenen Erlebens und des Wahrgenommenen durch Bezugnahme auf die Theorie» (Schweizer Charta, 2016, Abs. 1.8), wodurch Objektivität und Intersubjektivität zustande kommen. Objektivierung des intersubjektiven therapeutischen Beziehungsgeschehens durch Reflexion im intersubjektiven Prozess der Supervision ermöglicht die Erfüllung des allgemeinen Wissenschaftskriteriums der Intersubjektivität.

2) Grundsätzlich geht es in der Psychotherapie «um das hermeneutische Erfassen der Individualität des Seelischen, das subjektive Erleben des Patienten" (Wagner, 1996, S. 244). Dennoch kommt die Psychotherapieforschung in ihrer Theoriebildung nicht um Verallgemeinerung herum. Um der Individualität des einzelnen Subjekts und der Einzigartigkeit des therapeutischen Beziehungsgeschehens gerecht zu werden, hat die Psychotherapieforschung von einzelnen Fallbeispielen auszugehen, um im Vergleich dieser Einzelfälle Muster oder Regularitäten aufzufinden. Diese Muster haben einen allgemeinen Charakter und sind als qualitative therapeutische Erfahrung formulierbar. Das Spannungsverhältnis zwischen vorurteilsfreiem, die Individualität des einzelnen fokussierendem Verstehen und dem Wissen um allgemeinere Zusammenhänge und Muster ist für Wagner (ebd., S. 235) charakteristisch für die Psychotherapie: «In der konkreten Behandlungssituation vollzieht sich der hermeneutische Zirkel zwischen Fragen, die dem Verständnis der Individualität des einzelnen dienen, und der Reflexion dieser Informationen vor dem theoretischen Hintergrund der jeweiligen psychotherapeutischen Theorie.»

Gegenüber der objektivierenden Psychologie und der am Verhalten orientierten Psychiatrie, die messbare und quantitative Methoden zur Erforschung der Psyche des Menschen verwenden, geht es der Psychotherapie um die Einzigartigkeit des Subjekts und des therapeutischen Beziehungsgeschehens. Sowohl die Berücksichtigung des «spezifisch Persönlichen", das heisst der Individualität, als auch der Einbezug des Subjektiven erfordern die Verwendung der qualitativen Forschungsmethode der Selbstreflexion.

3) Eine Möglichkeit, Widersprüche nicht einfach zu eliminieren, sondern für den Erkenntnisprozess fruchtbar zu machen, ist die dialektische Methode. Allerdings gibt es keine weithin anerkannte Formulierung dieser Denkweise, sondern eine Vielzahl von Ansätzen.

Der Grund, weshalb die dialektische Methode für die Psychotherapie von Interesse ist, ist die Bedeutung des Beziehungsgeschehens in der therapeutischen Situation, «in der Subjekt und Objekt nicht getrennt betrachtet werden können» (Schweizer Charta, 2016, Abs. 1.8). In dieser Situation «stehen die Therapierenden in einer unaufhebbaren Dialektik zwischen Begegnung und Objektivierung der Klientel und ihrer selbst» (ebd.). Mit der «unaufhebbaren Dialektik» ist eine nicht eliminierbare Widersprüchlichkeit bzw. Ambivalenz der therapeutischen Beziehung gemeint: TherapeutIn und KlientIn sind zugleich sowohl Subjekte eines intersubjektiven Geschehens als auch Objekte ihrer selbst in der Selbstreflexion auf das subjektive Erleben des intersubjektiven Geschehens.

Die Dialektik des Selbstbewusstseins von Fischer (2008, S. 124ff.) stellt einen Ansatz dar, der speziell für die Psychotherapie bzw. die Psychotherapiewissenschaft ausgearbeitet und mittels der Kategorien Subjektivität, Objektivität und Intersubjektivität formuliert worden ist. Dabei entspricht der Subjektivität das unmittelbare Selbsterleben, das «subjektive Selbst», der Objektivität das Bewusstsein, das das Subjekt von seinem Selbst hat, und der Intersubjektivität das Selbstbewusstsein, das sowohl das subjektive als auch das objektive Selbst umfasst. Eine dialektische Psychotherapie geht von der Anerkennung objektiver Widersprüche bzw. Antinomien aus, die Krisen zugrunde liegen und deren Überwindung psychogenetische Entwicklungsschritte bedeuten (vgl. Kesselring, 1984, S. 18f.). So hat Fischer (2008) mit Bezug auf Wandschneider (1995) eine dialektische Methode entworfen, in der die Antinomie die Ausgangslage für die Kategorienentwicklung bildet.

Zusammenfassend lässt sich sagen, dass die Konkordanz des Wissenschaftsbegriffs der Psychotherapiewissenschaft mit dem allgemeinen Wissenschaftsbegriff davon abhängt, inwieweit 1) (Selbst-)Reflexion als qualitative Methode der Erkenntnis von (Inter-)Subjektivität, 2) Individualität als eine trotz Generalisierung zu bewahrende psychische Dimension, sowie 3) alogische Strukturen wie Widersprüche, Antinomien und Ambivalenzen als produktive Entwicklungsfaktoren anerkannt werden. 


\section{Das Selbstverständnis der Psychotherapie- wissenschaft als Wissenschaft und ihr Verhältnis zur Medizin, Psychologie und Psychiatrie}

Die Psychotherapiewissenschaft sieht sich gemäss Schweizer Charta (2016, Abs. 1.8) als «Schwesterdisziplin der Psychiatrie und Psychologie». Während sich die Psychiatrie (als Teildisziplin der Medizin) und die Psychologie hinsichtlich verwendeter Methoden in Forschung, Theoriebildung und Therapie überwiegend an den Naturwissenschaften orientieren, integriert die Psychotherapiewissenschaft Erkenntnisse «verschiedener Wissensgebiete der Natur-, Kultur- und Geisteswissenschaften» in ihr eigenes Verständnis und ihre Konzepte. Während die Schweizer Gesetzgebung sie als blosses Anwendungsgebiet der Psychologie und der Medizin betrachtet, versteht sie sich als eigenständige wissenschaftliche Disziplin.

Medizin, Psychiatrie und klinische Psychologie orientieren sich weitgehend an den Methoden der Naturwissenschaft, von denen sie auch ihr Wissen beziehen. Dem entsprechen eine empirisch-kausalgenetische Vorgehensweise in der medizinischen Forschung und die Verwendung technischer Verfahren zur Diagnose und Behandlung von Krankheiten. Sowohl die dabei stattfindende «Reduktion des Organismus auf die materielle Dimension» als auch die Fokussierung auf «objektivierbare Eigenschaften des kranken Organismus» verhindern das Erfassen der seelischen Individualität. Dagegen geht es in der Psychotherapiewissenschaft um das «hermeneutische Erfassen der Individualität des Seelischen» (Wagner, 1996, S. 244).

Im Unterschied zu Medizin, Psychiatrie und Psychologie steht in der Psychotherapiewissenschaft das Beziehungsgeschehen zwischen KlientIn und TherapeutIn im Zentrum sowohl des Heilverfahrens als auch der Forschungsarbeit. Die Psychotherapiewissenschaft ist eine «dialogische Wissenschaft», die im Grunde als «eine Subjekt-Subjekt-Beziehung konstituiert ist, bei der aus dem Dialog eine intersubjektive Bedeutungsstruktur entsteht» (ebd., S. 230). Die Dialogfähigkeit ist daher wichtigste psychotherapeutische Kompetenz, denn die TherapeutInnen sind «selbst als Dialogpartner Bestandteil des therapeutischen Prozesses», wohingegen SchulmedizinerInnen und klinische PsychologInnen «sich mittels Diagnostik und Verschreibung von diesem Dialog distanzieren» (ebd., S. 231).

Ziel des psychotherapeutischen Heilverfahrens sowohl seitens der PatientInnen als auch seitens der TherapeutInnen ist zunächst «die Selbsterkenntnis, sei es nun im Verständnis der eigenen Lebensgeschichte, der Beziehungskonstellationen, des Verhältnisses von Körperlichkeit mit allen psychosomatischen Problemen, der Analyse der Lebensziele und anderer Aspekte der Persönlichkeit in ihrem psychosozialen Kontext» (Pritz \& Teufelhart, 1996, S. 3) mittels Selbstreflexion. Ziel der psychotherapeutischen Forschungsarbeit ist ausgehend von der therapeutischen Praxis die Theoriebildung durch schrittweise Generalisierung des konkreten, individuellen Beziehungsgeschehens mittels Reflexion und Abstraktion.
Durch diesen vielschichtigen, gemeinsamen Reflexionsprozess von PatientIn und TherapeutIn unterscheidet sich die Psychotherapiewissenschaft grundsätzlich von der Medizin, der Psychologie und der Psychiatrie. Er schliesst sowohl die Selbstreflexion auf das subjektive Erleben des intersubjektiven Beziehungsgeschehens als auch die Reflexion auf ausserhalb der therapeutischen Situation liegende Lebensbereiche ein und bildet eine wesentliche Dimension des therapeutischen Prozesses. Die Auffassung der Psychotherapie als blosses Anwendungsgebiet von Medizin und Psychologie greift daher zu kurz.

Fazit: Es gibt meines Erachtens keinen Grund, der Psychotherapiewissenschaft einen anderen wissenschaftlichen Status zuzuschreiben als der Medizin, die ihr Wissen vorwiegend aus anderen wissenschaftlichen Disziplinen insbesondere den naturwissenschaftlichen - bezieht und anwendet. Medizin und Psychotherapiewissenschaft sind beide angewandte Wissenschaften von Heilverfahren, deren Absicht die Förderung körperlicher und seelischer Gesundheit ist. Das Spektrum der Wissenschaften, aus denen die Psychotherapiewissenschaft ihr Wissen bezieht, ist aber ein anderes als dasjenige der Medizin oder der Psychiatrie. Die Medizin betrifft zunächst das Leibliche von Lebewesen, die Psychiatrie als Teilgebiet der Medizin und die Psychotherapiewissenschaft betreffen den Menschen in seiner seelisch-leiblichen Ganzheit, allerdings aus unterschiedlichen Perspektiven und mittels unterschiedlicher Methoden.

Ein Charakteristikum von Wissenschaft ist die Wissensgenerierung: «Um von Wissenschaft sprechen zu können, muss mit einer angebbaren Methode (Wissen geschaffen» werden» (Wagner, 1996, S. 223). Wie die medizinische Forschung für die Medizin generiert auch die Psychotherapieforschung spezifisches Wissen, das in der psychotherapeutischen Praxis Anwendung findet. In der Psychotherapiewissenschaft ist der gemeinsame, dialogische Reflexionsprozess von PatientIn und TherapeutIn Ort des therapeutischen Heilens und der Wissensgenerierung. Die Methode der Reflexion macht die Eigenständigkeit und Wissenschaftlichkeit der Psychotherapiewissenschaft aus.

\section{Literatur}

Barwinski, R. (2017). Intersubjektivität und Selbstreflexion. Psychotherapie-Wissenschaft, 7(1), 23-30.

Buchmann, R., Schlegel, M. \& Vetter, J. (1996). Die Eigenständigkeit der Psychotherapie in Wissenschaft und Praxis. In A. Pritz (Hrsg.), Psychotherapie - eine neue Wissenschaft vom Menschen (S. 75121). Wien: Springer.

Diemer, A. (1964). Was heisst Wissenschaft? Meisenheim am Glan: Verlag Anton Hain.

Fischer, G. (2008). Logik der Psychotherapie. Philosophische Grundlagen der Psychotherapiewissenschaft. Kröning: Asanger.

Frank, M. (1991). Selbstbewusstsein und Selbsterkenntnis. Stuttgart: Reclam.

Kesselring, T. (1984). Die Produktivität der Antinomie. Frankfurt/M: Suhrkamp.

Pritz, A. \& Teufelhart, H. (1996). Psychotherapie - Wissenschaft vom Subjektiven. In A. Pritz (Hrsg.), Psychotherapie-eine neue Wissenschaft vom Menschen (S. 1-18). Wien: Springer. 
v. Uexküll, J. (1931). Die Rolle des Subjekts in der Biologie. Naturwissenschaften, 19, 386-391.

Verein Schweizer Charta für Psychotherapie (Hrsg.). (2016). Schweizer Charta für Psychotherapie. Zürich: Verein Schweizer Charta für Psychotherapie.

Wagner, E. (1996). Psychotherapie als Wissenschaft in Abgrenzung von der Medizin. In A. Pritz (Hrsg.), Psychotherapie - eine neue Wissenschaft vom Menschen (S. 219-147). Wien: Springer.

Wandschneider, D. (1995). Grundzüge einer Theorie der Dialektik. Stuttgart: Klett.

Wohlgenannt, R. (1969). Was ist Wissenschaft? Braunschweig: Friedr. Vieweg \& Sohn.

\section{The Scientific Concept of Psychotherapy Studies}

A scientific practice without a scientific concept is possible. However, the scientific concept of a particular science constitutes and clarifies the self-understanding of this science. The purpose of my article is the presentation and discussion of the concept of psychotherapy science. This deviates from the generally accepted concept of science: it involves the subjective, focuses on the individual and recognizes contradictions and ambivalences as factors of scientific knowledge. I want to show how, in spite of these deviations, a correspondence between the concept of psychotherapy and the generally recognized conception of science is possible.

Key words: concept of science, psychotherapy science, the subjective, the individual, the contradictory

\section{Il concetto scientifico della psicoterapia scientifica}

Una pratica scientifica è certamente possibile senza un concetto scientifico. Il concetto scientifico di una particolare scienza, tuttavia, costituisce e chiarisce l'autocomprensione di questa scienza. Lo scopo del mio articolo è la presentazione e la discussione del concetto di psicoterapia scientifica. Ciò si discosta dal concetto generalmente accettato di scienza: include il soggettivo, pone l'attenzione sull'individuale e riconosce le contraddizioni e le ambivalenze come fattori della conoscenza scientifica. Voglio mostrare come, nonostante queste discrepanze, sia possibile una corrispondenza tra il concetto scientifico di psicoterapia scientifica e la concezione generalmente accettata di scienza.

Parole chiave: concetto scientifico, psicoterapia scientifica, il soggettivo, l'individuale, il contraddittorio

\section{Der Autor}

Markus Erismann, Dr. phil., studierte von 1983 bis 1989 Philosophie und Germanistik an der Universität Zürich und promovierte 1995/96 am Philosophischen Seminar der Universität Zürich. Seine Arbeitsschwerpunkte sind Erkenntnistheorie und Methodologie.

\section{Kontakt}

Dr. Markus Erismann

Säntisstrasse 2, 8008 Zürich

E-Mail: markus.erismann@bluewin.ch 